\title{
An annotated checklist of opisthobranch fauna (Gastropoda: Opisthobranchia) of the Nicobar Islands, India
}

\author{
C.R. Sreeraj ${ }^{1}$, C. Sivaperuman ${ }^{2} \&$ C. Raghunathan ${ }^{3}$ \\ 1,2,3 Zoological Survey of India, Andaman and Nicobar Regional Centre, National Coral Reef Researh Institute, Port Blair, Andaman \\ and Nicobar Islands 744102, India \\ Email: ${ }^{\mathrm{c}} \mathrm{crsreeraj@gmail.com} \mathrm{(corresponding} \mathrm{author),}{ }^{2}$ __sivaperuman@yahoo.com, ${ }^{3}$ raghuksc@rediffmail.com
}

\begin{abstract}
This paper presents 52 species of opisthobranchs recorded from the Nicobar group of Islands. Of these, Aldisa erwinkoehleri, Dermatobranchus rodmani, Glossodoris pallida, Noumea simplex, Pectenodoris trilineata, Okenia kendi, Tambja morosa, Phyllidia elegans, Phyllidiopsis annae, Flabellina riwo and Phidiana indica represent new records for Indian waters.
\end{abstract}

Keywords: India, opisthobranch, Nicobar, nudibranch.

The Andaman and Nicobar archipelago consists of 572 islands, islets and rocky outcrops with an aggregate coastline of $1,962 \mathrm{~km}$. The continental shelf area is very limited with an estimated area of $16,000 \mathrm{~km}^{2}$ and the sea is very deep within a few kilometers from the shore. The Exclusive Economic Zone (EEZ) around the islands encompasses around 0.6 million $\mathrm{km}^{2}$, which is again around $30 \%$ of the EEZ of India. This provides a great opportunity to explore the vast diversity of the

\author{
Date of publication (online): 26 April 2012 \\ Date of publication (print): 26 April 2012 \\ ISSN 0974-7907 (online) | 0974-7893 (print) \\ Editor: Deepak Apte \\ Manuscript details: \\ Ms \# 02783 \\ Received 03 April 2011 \\ Final received 05 January 2012 \\ Finally accepted 27 February 2012
}

Citation: Sreeraj, C.R., C. Sivaperuman \& C. Raghunathan (2012). An annotated checklist of opisthobranch fauna (Gastropoda: Opisthobranchia) of the Nicobar Islands, India. Journal of Threatened Taxa 4(4): 24992509 .

Copyright: @ C C.R. Sreeraj, C. Sivaperuman \& C. Raghunathan 2012. Creative Commons Attribution 3.0Unported License. JoTT allows unrestricted use of this article in any medium for non-profit purposes, reproduction and distribution by providing adequate credit to the authors and the source of publication.

Acknowledgements: The authors are thankful to Dr. K. Venkataraman, the Director, Zoological Survey of India for the facilities provided. The financial assistance supported by the Ministry of Environment and Forests, Government of India is also acknowledged.

\section{OPEN ACCESS | FREE DOWNLOAD}

seas around these islands. Opisthobranchs are one of the less studied groups of molluscs in Andaman and Nicobar Islands.

The earliest opisthobranch study in Indian waters dates back to 1864 with the work of Alder \& Hancock. The knowledge about opisthobranchiate faunal diversity of Indian subcontinent is too little to interpret. Although variegated, these organisms could drag the attention of only a few scientists. In recent times Apte (2009), Apte et al. (2010), Raghunathan et al. (2010), Ramakrishna et al. (2010), Sreeraj et al. (2010), Apte \& Salahuddin (2011), and Matwal \& Joshi (2011) studied opisthobranch fauna of India.

The molluscan studies of Andaman and Nicobar Islands were started in the late $19^{\text {th }}$ century. The available literature shows that the earliest molluscan study was on a collection of marine shells made by E.A. Smith in 1878. The first report on nudibranch from these Islands was published by Eliot (1910), which deals with a collection of nudibranchs by Annandale. Opisthobranchiate taxonomy and ecology of these Islands has recently commenced (Raghunathan et al. 2010; Ramakrishna et al. 2010; Sreeraj et al. 2010) including the discovery of many new records for the Indian subcontinent.

There has been no research on the community structure and population dynamics of opisthobranchs from Andaman and Nicobar Islands. Quantitative studies on opisthobranch populations in Indian waters are scarce. This is due to opisthobranchs' inherent low numerical density; individuals are small and often cryptic, and mostly sub-tidal. No studies are available on the opisthobranchs of the Nicobar group of Islands; therefore the present attempt has been made to compile the list on the occurrence of this group of animals based on the field surveys conducted during 2009-2011. 


\section{Materials and Methods}

The Nicobar Islands are situated in the south-east of the Bay of Bengal between $6-10^{\circ} \mathrm{N}$ and $92-94^{\circ} \mathrm{E}$. There are altogether 22 large and small islands, out of which only 12 are inhabited. The most northerly island of the group is Car Nicobar, which is $225 \mathrm{~km}$ from Port Blair and the ten degree channel (about 120km wide) separates this Island from Little Andaman. Chowra, Teressa, Bompoka, Katchal, Kamorta, Nancowry and Trinket form the central group of Nicobar Islands; while in the southern group are Pulo Milo, Little Nicobar, Kondul, and Great Nicobar. The extreme southern point of Great Nicobar, previously known as Pygmalion Point and now Indira Point, is about $145 \mathrm{~km}$ from Pulo Brass of Achin Head of Sumatra. The uninhabited islands in the central and southern groups are Batti Malv, Tileangchong and Meroe, Trak, Treis, Menchal and Kabra, respectively.

Survey sites were selected based on the habitat features and accessibility (Fig 1). Sampling was carried out primarily by scuba diving up to a depth of $30 \mathrm{~m}$. Most of the specimens were measured and photographed in their natural habitat and collected in a plastic jar before being brought to the laboratory for examination. Animals were fixed in a solution of $5 \%$ formaldehyde and seawater. Before placement in the fixative solution the animals were narcotised with a solution consisting of $72 \mathrm{~g} / 1$ of $\mathrm{MgCl}_{2}$. The formaldehyde fixed animals were transferred to $95 \%$ ethanol for long term preservation. All the collected specimens are deposited in the National Zoological Collections of the Zoological Survey of India, Port Blair.

Identification was carried out based on the external morphology only and using following literature; Gosliner et al. (2008), Rudman (1982, 1983, 1984, 1986, 1995), and Brunckhorst (1993); and two webbased portals, the Australian Museum's Seaslug Forum (http://www.seaslugforum.net/) and Nudi Pixel (http:// www.nudipixel.net). Taxonomic changes published recently are incorporated.

Many of these species have wider distribution globally. However, in the present paper we have provided its occurrence in Indian waters only.

\section{Results and Discussion}

A total of 161 specimens belonging to 52 species were recorded during the study period. These belong to three orders, namely; Cephalaspidea (four species), Sacoglossa (four species), and Nudibranchia (44 species). The Nudibranchia was the most dominant taxon in this study. The distribution of the species

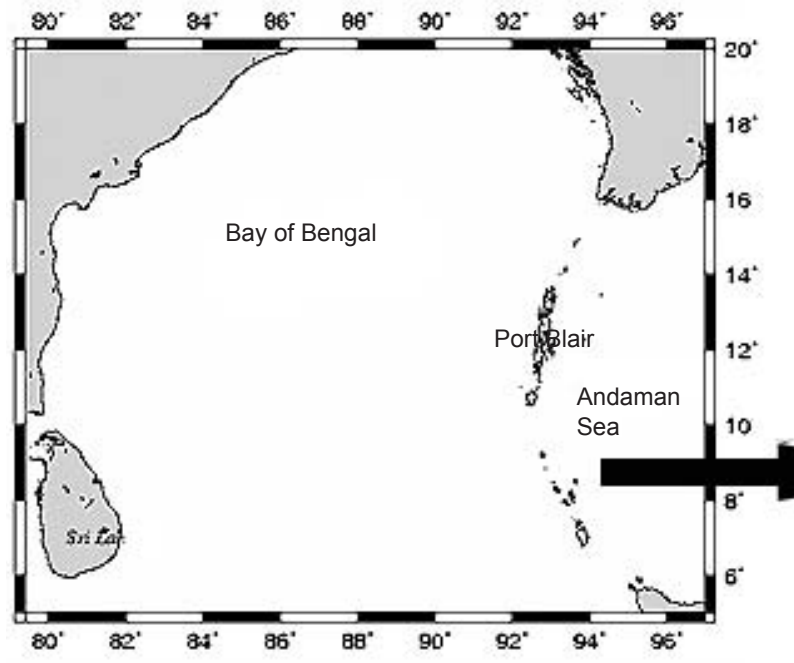

Figure 1. Nicobar Islands.

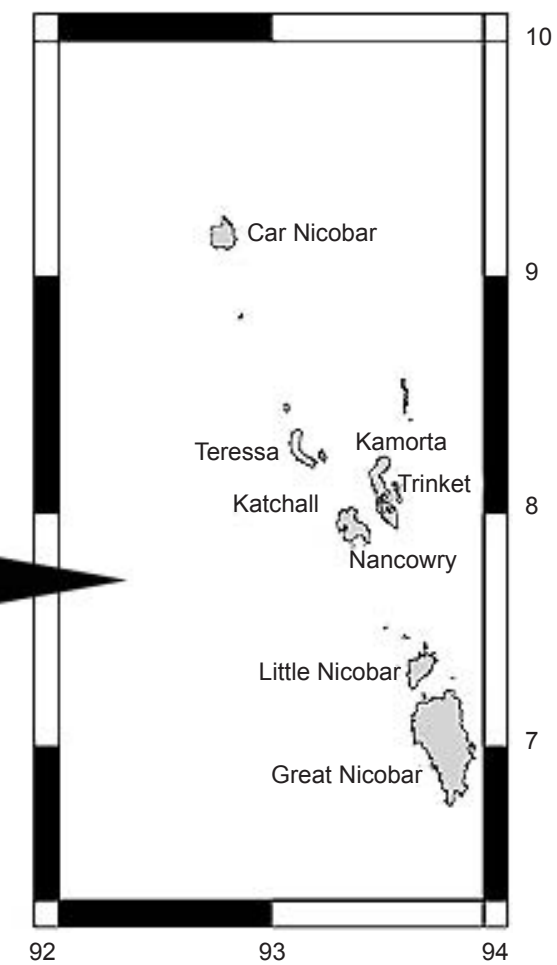


along different islands of Nicobar group is presented in Table 1. A systematic account of the species recorded is given below and detailed descriptions have been given only for the newly reported species.

\section{Cephalaspidea Fischer, 1883 \\ Bullidae Gray, 1827}

1. Bulla ampulla Linnaeus, 1758. Material examined: 16.ii.2011, two specimens, Champion, Nancowry Island. Distribution in India: All along the west and east coast including Lakshadweep and Andaman Islands (Satyamurti 1952; Rao \& Dey 2000; Rao 2003; Apte 2009).

\section{Haminoeidae Pilsbry, 1895}

2. Atys naucum (Linnaeus, 1758). Material examined: 16.ii.2011, one specimen, Champion, Nancowry Island. Identification: Shell is thin and globose. The spire is deeply submerged within the body whorl. Shell is smooth in appearance superficially. Faint growth lines cross the spiral grooves. (Image 1). Distribution in India: Andaman Islands (Rao \& Dey 2000; Rao 2003).

\section{Aglajidae Pilsbry, 1895}

3. Chelidonura punctata Eliot, 1903. Material examined: 19.xi.2009, five specimens, Car Nicobar. Distribution in India: Andaman and Lakshadweep Islands (Apte 2009; Ramakrishna et al. 2010).

4. Philinopsis gardineri (Eliot, 1903). Material examined: 21.ii.2011, two specimens, Safed balu, Trinket Island. 01.i.2010, one specimen, Campbell Bay, Great Nicobar. Distribution in India: Andaman Islands.

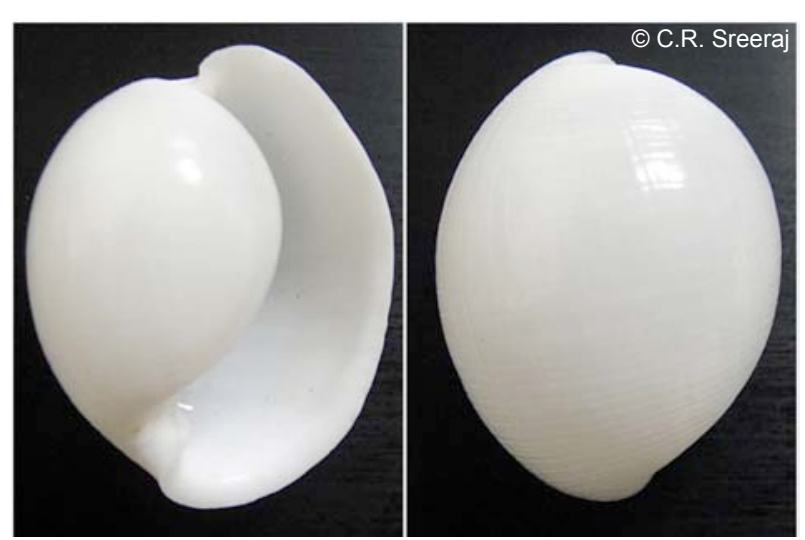

Image 1. Atys naucum
Sacoglossa (von lhering, 1876)

Plakobranchidae Gray, 1840

5. Elysia pusilla Bergh, 1872. Material examined: 12.viii.2010, two specimens, Car Nicobar. Distribution in India: Nicobar Islands.

6. Plakobranchus ocellatus Van Hasselt, 1824. Material examined: 12.viii.2010, four specimens, Car Nicobar. Distribution in India: Andaman Islands, Tamil Nadu, Lakshadweep Islands (Rao 1961; Apte 2009; Ramakrishna et al. 2010).

7. Thuridilla cf. bayeri (Er. Marcus, 1965). Material examined: 28.ii.2010, two specimens, Kamorta Island; 21.ii.2011, one specimen, Safed Balu, Trinket Island. Distribution in India: Andaman Islands (Ramakrishna et al. 2010)

8. Thuridilla moebii (Bergh, 1888). Material examined: 28.ii.2010, three specimens, Kamorta Island; 20.ii.2011, three specimens, Kapang, Katchall Island; 21.ii.2011, two specimens, Safed Balu, Trinket Island. Distribution in India: Andaman Islands.

\section{Nudibranchia Blainville, 1814 \\ Hexabranchidae Bergh, 1881}

9. Hexabranchus sanguineus (Rüppell \& Leuckart, 1830). Material examined: 12 .viii.2010, One specimen, Car Nicobar. Distribution in India: Andaman Islands, Lakshadweep Islands, Kerala (Eliot 1906; Narayanan 1968; Narayanan 1970; Ramakrishna et al. 2010; Apte \& Salahuddin 2011)

\section{Polyceridae Alder \& Hancock, 1845 Nembrothinae Burn, 1967}

10. Tambja morosa (Bergh, 1877). Material examined: 19.viii.2011, three specimens, Kapila, Trinket Island; 22.ii.2011, three specimens, Kamorta Jetty. Identification: Body dark blue or more commonly black with blue markings on the head, notum and foot. Rhinophores and gills black (Image 2). Natural history: A common inhabitant of muddy bottomed reefs and vertical substrata like wharfs where it is found on arborescent bryozoans. Comparatively a fast moving nudibranch. Distribution in India: Nicobar Islands, a new record from the present study. Remarks: New record for Indian waters.

\section{Goniodorididae H. Adams \& A. Adams, 1854}

11. Okenia kendi Gosliner, 2004. Material examined: 20.ii.2011, one specimen, Kapang, Katchall 
Table 1. Distribution data of opisthobranchs in various Islands of Nicobar group.

\begin{tabular}{|c|c|c|c|c|c|c|c|}
\hline & Species & $\mathrm{CN}$ & KM & NC & TK & KL & GN \\
\hline 1 & $\begin{array}{l}\text { Bulla ampulla } \\
\text { Linnaeus, } 1758\end{array}$ & & & + & & & \\
\hline 2 & $\begin{array}{l}\text { Atys naucum } \\
\text { (Linnaeus, 1758) }\end{array}$ & & & + & & & \\
\hline 3 & $\begin{array}{l}\text { Chelidonura } \\
\text { punctata Eliot, } 1903\end{array}$ & + & & & & & \\
\hline 4 & $\begin{array}{l}\text { Philinopsis gardineri } \\
\text { (Eliot, 1903) }\end{array}$ & & & & + & & \\
\hline 5 & $\begin{array}{l}\text { Elysia pusilla Bergh, } \\
1872\end{array}$ & + & & & & & \\
\hline 6 & $\begin{array}{l}\text { Plakobranchus } \\
\text { ocellatus Van } \\
\text { Hasselt, } 1824\end{array}$ & + & & & & & + \\
\hline 7 & $\begin{array}{l}\text { Thuridilla cf bayeri } \\
\text { (Marcus, 1965) }\end{array}$ & & & & + & & \\
\hline 8 & $\begin{array}{l}\text { Thuridilla moebii } \\
\text { (Bergh, 1888) }\end{array}$ & & & & + & + & \\
\hline 9 & $\begin{array}{l}\text { Hexabranchus } \\
\text { sanguineus (Rüppell } \\
\text { \& Leuckart, 1828) }\end{array}$ & + & & & & & \\
\hline 10 & $\begin{array}{l}\text { Tambja morosa } \\
\text { (Bergh, 1877) }\end{array}$ & & + & & + & & \\
\hline 11 & $\begin{array}{l}\text { Okenia kendi } \\
\text { Gosliner, } 2004\end{array}$ & & & & & + & \\
\hline 12 & $\begin{array}{l}\text { Gymnodoris citrina } \\
\text { (Bergh, 1875) }\end{array}$ & & & & & + & \\
\hline 13 & $\begin{array}{l}\text { Gymnodoris } \\
\text { impudica (Rüppell \& } \\
\text { Leuckart, 1828) }\end{array}$ & & + & & & & \\
\hline 14 & $\begin{array}{l}\text { Aldisa erwinkoehleri } \\
\text { Perrone, } 2001\end{array}$ & & + & & & & \\
\hline 15 & $\begin{array}{l}\text { Discodoris } \\
\text { boholiensis Bergh, } \\
1877\end{array}$ & & & + & & & \\
\hline 16 & $\begin{array}{l}\text { Halgerda tessellata } \\
\text { (Bergh, 1880) }\end{array}$ & & & & & + & \\
\hline 17 & $\begin{array}{l}\text { Jorunna funebris } \\
\text { (Kelaart, 1858) }\end{array}$ & & & & & & + \\
\hline 18 & $\begin{array}{l}\text { Chromodoris } \\
\text { conchyliata Yonow, } \\
1984\end{array}$ & & + & & & & \\
\hline 19 & $\begin{array}{l}\text { Chromodoris } \\
\text { elisabethina Bergh, } \\
1877\end{array}$ & & & & + & & \\
\hline 20 & $\begin{array}{l}\text { Chromodoris fidelis } \\
\text { (Kelaart, 1858) }\end{array}$ & & + & + & + & & \\
\hline 21 & $\begin{array}{l}\text { Chromodoris } \\
\text { geometrica (Risbec, } \\
1928 \text { ) }\end{array}$ & & + & & + & & \\
\hline 22 & $\begin{array}{l}\text { Glossodoris } \\
\text { atromarginata } \\
\text { (Cuvier, 1804) } \\
\end{array}$ & + & & + & & & \\
\hline 23 & $\begin{array}{l}\text { Glossodoris pallida } \\
\text { (Rüppell \& Leuckart, } \\
\text { 1830) }\end{array}$ & & + & & & & \\
\hline 24 & $\begin{array}{l}\text { Hypselodoris } \\
\text { bullockii } \\
\text { (Collingwood, 1881) }\end{array}$ & & & & + & & \\
\hline 25 & $\begin{array}{l}\text { Hypselodoris } \\
\text { maculosa (Pease, } \\
\text { 1871) }\end{array}$ & & + & & + & & \\
\hline 26 & $\begin{array}{l}\text { Hypselodoris } \\
\text { maridadilus } \\
\text { Rudman, } 1977 \\
\end{array}$ & & + & + & & & \\
\hline
\end{tabular}

\begin{tabular}{|c|c|c|c|c|c|c|c|}
\hline 27 & $\begin{array}{l}\text { Hypselodoris } \\
\text { nigrostriata (Eliot, } \\
1904 \text { ) }\end{array}$ & & & & + & & \\
\hline 28 & $\begin{array}{l}\text { Noumea simplex } \\
\text { (Pease, 1871) }\end{array}$ & & & & & + & \\
\hline 29 & $\begin{array}{l}\text { Pectenodoris } \\
\text { trilineata (Adams \& } \\
\text { Reeve, 1850) }\end{array}$ & & & & & + & \\
\hline 30 & $\begin{array}{l}\text { Risbecia } \\
\text { ghardaqana (Gohar } \\
\text { \& Aboul-Ela, 1957) }\end{array}$ & & & & + & & \\
\hline 31 & $\begin{array}{l}\text { Risbecia pulchella } \\
\text { (Rüppell \& Leuckart, } \\
\text { 1828) }\end{array}$ & & + & & & & \\
\hline 32 & $\begin{array}{l}\text { Thorunna australis } \\
\text { (Risbec, 1928) }\end{array}$ & & + & + & + & + & \\
\hline 33 & $\begin{array}{l}\text { Thorunna florens } \\
\text { (Baba, 1949) }\end{array}$ & & & & & + & \\
\hline 34 & $\begin{array}{l}\text { Thorunna horologia } \\
\text { Rudman, } 1984\end{array}$ & & & & & + & \\
\hline 35 & $\begin{array}{l}\text { Phyllidia marindica } \\
\text { (Yonow \& Hayward, } \\
\text { 1991) }\end{array}$ & & & + & + & + & + \\
\hline 36 & $\begin{array}{l}\text { Phyllidia coelestis } \\
\text { Bergh, } 1905\end{array}$ & & + & & & & \\
\hline 37 & $\begin{array}{l}\text { Phyllidia elegans } \\
\text { Bergh, } 1869\end{array}$ & & & & + & & \\
\hline 38 & $\begin{array}{l}\text { Phyllidia varicosa } \\
\text { Lamarck, } 1801\end{array}$ & + & + & & + & + & \\
\hline 39 & $\begin{array}{l}\text { Phyllidiella pustulosa } \\
\text { (Cuvier, 1804) }\end{array}$ & & + & & & & \\
\hline 40 & $\begin{array}{l}\text { Phyllidiella rosans } \\
\text { (Bergh, 1873) }\end{array}$ & & + & & & & \\
\hline 41 & $\begin{array}{l}\text { Phyllidiella rudmani } \\
\text { Brunckhorst, } 1993\end{array}$ & + & + & & + & + & \\
\hline 42 & $\begin{array}{l}\text { Phyllidiella zeylanica } \\
\text { (Kelaart, 1859) }\end{array}$ & & + & & + & + & + \\
\hline 43 & $\begin{array}{l}\text { Phyllidiopsis annae } \\
\text { Brunckhorst, } 1993\end{array}$ & & + & & & & \\
\hline 44 & $\begin{array}{l}\text { Phyllidiopsis } \\
\text { gemmata (Pruvot- } \\
\text { Fol, 1957) }\end{array}$ & & & & + & & \\
\hline 45 & $\begin{array}{l}\text { Phyllidiopsis } \\
\text { phiphiensis } \\
\text { Brunckhorst, } 1993\end{array}$ & & & & + & & \\
\hline 46 & $\begin{array}{l}\text { Phyllidiopsis striata } \\
\text { Bergh, } 1888\end{array}$ & & & & + & & \\
\hline 47 & $\begin{array}{l}\text { Dermatobranchus } \\
\text { rodmani Baba, } 1949\end{array}$ & & + & & & & \\
\hline 48 & $\begin{array}{l}\text { Flabellina bicolor } \\
\text { (Kelaart, 1858) }\end{array}$ & & & & & + & \\
\hline 49 & $\begin{array}{l}\text { Flabellina riwo } \\
\text { Gosliner \& Willan, } \\
1991\end{array}$ & & & & & + & \\
\hline 50 & $\begin{array}{l}\text { Flabellina } \\
\text { rubrolineata } \\
\text { (O'Donoghue, 1929) }\end{array}$ & & & + & 0 & & \\
\hline 51 & $\begin{array}{l}\text { Phidiana indica } \\
\text { (Bergh, 1896) }\end{array}$ & & & + & & + & \\
\hline \multirow[t]{2}{*}{52} & $\begin{array}{l}\text { Pteraeolidia ianthina } \\
\text { (Angas, } 1864 \text { ) }\end{array}$ & & & + & & & \\
\hline & Total & 7 & 19 & 11 & 21 & 16 & 4 \\
\hline
\end{tabular}

CN - Car Nicobar; KM - Kamorta Island; NC - Nancowry Island; TK Trinket Island; KL - Katchall Island; GN - Great Nicobar Island 


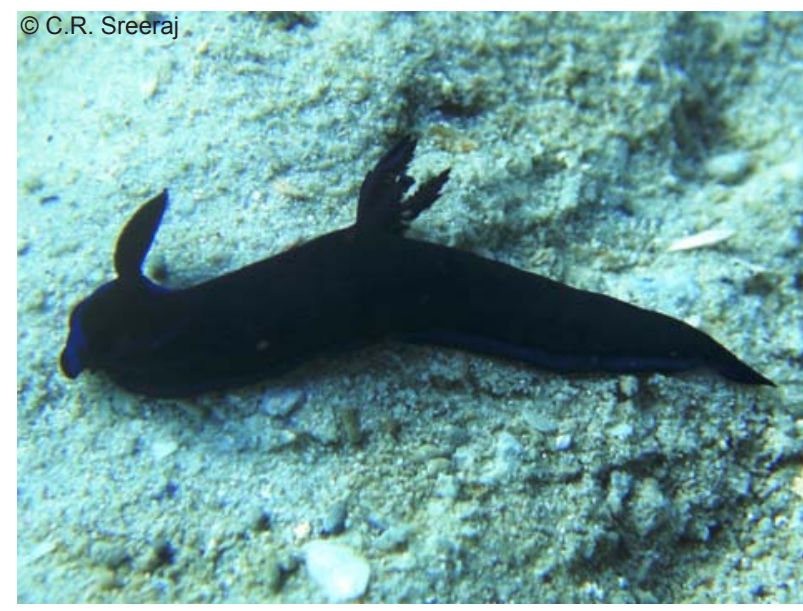

Image 2. Tambja morosa

Island. Identification: It can be identified by its white body with brown and purple pigment on the back and slender appendages (Image 3). Natural history: Found on the rocks with sponges, where it feeds on encrusting bryozoans. Distribution in India: Nicobar Islands, a new record from the present study. Remarks: New record for the Indian Ocean.

\section{Gymnodorididae Odhner, 1941}

12. Gymnodoris citrina (Bergh, 1875). Material examined: 20.ii.2011, two specimens, Kapang, Katchall Island. Distribution in India: Andaman Islands (Ramakrishna et al. 2010). 13. Gymnodoris impudica (Rüppell \& Leuckart, 1828). Material examined: 15.xi.2009, one specimen, Kamorta Island. Distribution in India: Andaman Islands (Ramakrishna et al. 2010).

\section{Cadlinidae Bergh, 1891}

14. Aldisa erwinkoehleri Perrone, 2001. Material examined: 17.ii.2011, one specimen, Kardip, Kamorta Island. Identification: This species can be easily distinguished by its dark colored rhinophores and yellow pigment on the tubercles immediately posterior to the rhinophores (Image 4). Natural history: This is one among the three species of Aldisa which mimics Phyllidia coelestis. Distribution in India: Nicobar Islands, a new record from the present study. Remarks: Previously known only from Thailand.

\section{Discodorididae Bergh, 1891}

15. Discodoris boholiensis Bergh, 1877. Material

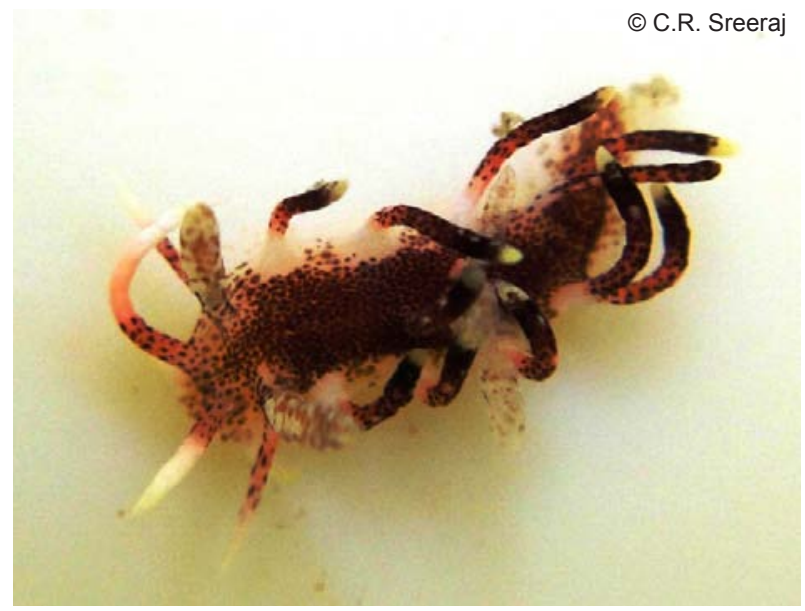

Image 3. Okenia kendi

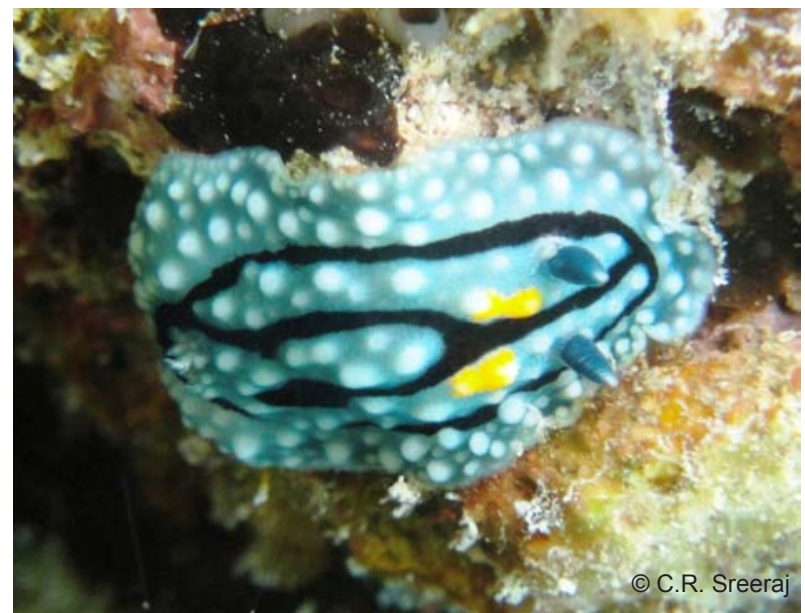

Image 4. Aldisa erwinkoehleri

examined: 16.ii.2011, one specimen, Champion, Nancowry Island. Distribution in India: Andaman Islands, Tamil Nadu, Gujarat (Rao 1960; Narayanan 1968; Dayrat 2010).

16. Halgerda tessellata (Bergh, 1880). Material examined: 20.ii.2011, four specimens, Kapang, Katchall Island. Distribution in India:Andaman Islands, Lakshadweep Islands (Apte 2009; Ramakrishna et al. 2010).

17. Jorunna funebris (Kelaart, 1858). Material examined: 12.ix.2010, one specimen, B. quarry, Campbell Bay, Great Nicobar. Distribution in India: Andaman Islands, Tamil Nadu, Lakshadweep Islands, Andhrapradesh, Kerala, Gujarat (Alder \& Hancock 1864; Eliot 1906; Narayanan 1968; Fontana et al. 2001; Apte 2009; Apte et al. 2010; Ramakrishna et al. 2010) 


\section{Chromodorididae Bergh, 1891}

18. Chromodoris conchyliata Yonow, 1984. Material examined:22.ii.2011, one specimen, Kamorta Jetty. Distribution in India: Andaman Islands

19. Chromodoris elisabethina Bergh, 1877. Material examined: 19.ii.2011, three specimens, Kapila, Trinket Island. Distribution in India: Andaman Islands (Ramakrishna et al. 2010).

20. Chromodoris fidelis (Kelaart, 1858). Material examined: 26.ii.2010, one specimen, Kamorta Island; 16.ii.2011, one specimen, Champion, Nancowry Island; 18.ii.2011, three specimens, Alukiya, Kamorta Island; 19.ii.2011, two specimens, Kapila, Trinket Island. Distribution in India: Andaman Islands, Tamil Nadu, Lakshadweep Islands (Eliot 1906; Apte 2009; Ramakrishna et al. 2010)

21. Chromodoris geometrica (Risbec, 1928). Material examined: 21.ii.2011, one specimen, Safed Balu, Trinket Island; 22.ii.2011, one specimen, Kamorta Jetty. Distribution in India: Andaman Islands.

22. Glossodoris atromarginata (Cuvier, 1804). Material examined: 19.xi.2009, one specimen, Car Nicobar; 16.ii.2011, one specimen, Champion, Nancowry Island. Distribution in India: Andaman Islands, Tamil Nadu, Kerala (Eliot 1906; Fontana et al. 1999; Ramakrishna et al. 2010)

23. Glossodoris pallida (Rüppell \& Leuckart, 1830). Material examined: 18.ii.2011, one specimen, Alukiya, Kamorta Island. Identification: It has a translucent white body with central opaque markings and a yellow marginal band. (Image 5). Natural history: Inhabits patch reefs, where it is found on the encrusting sponges which it feeds up on. Distribution in India: Nicobar Islands, a new record from the present study. Remarks: New record for Indian waters

24. Hypselodoris bullockii (Collingwood, 1881). Material examined: 19.ii.2011, six specimens, Kapila, Trinket Island. Distribution in India: Andaman Islands (Ramakrishna et al. 2010).

25. Hypselodoris maculosa (Pease, 1871). Material examined: 19.ii.2011, one specimen, Kapila, Trinket Island; 22.ii.2011, one specimen, Kamorta Jetty. Distribution in India: Andaman Islands, Lakshadweep Islands (Apte 2009; Ramakrishna et al. 2010).

26. Hypselodoris maridadilus Rudman, 1977. Material examined: 16.ii.2011, two specimens, Kamorta Jetty; 18.ii.2011, one specimen, Alukiya, Kamorta Island. Distribution in India: Lakshadweep Islands (Apte 2009)

27. Hypselodoris nigrostriata (Eliot, 1904). Material examined: 19.ii.2011, one specimen, Kapila, Trinket Island. Distribution in India: Andaman Islands (Ramakrishna et al. 2010)

28. Noumea simplex (Pease, 1871). Material examined: 20.ii.2011, one specimen, Kapang, Katchall Island. Identification: Body colour pink. Gill and rhinophores tipped with deep orange. The gills vibrate while crawling; a typical character of the genus (Image 6). Natural history: It is reported to feed on pink sponges (Gosliner et al. 2008). Distribution in India: Nicobar Islands, a new record from the present study. Remarks: New record for Indian waters

29. Pectenodoris trilineata (A. Adams \& Reeve,

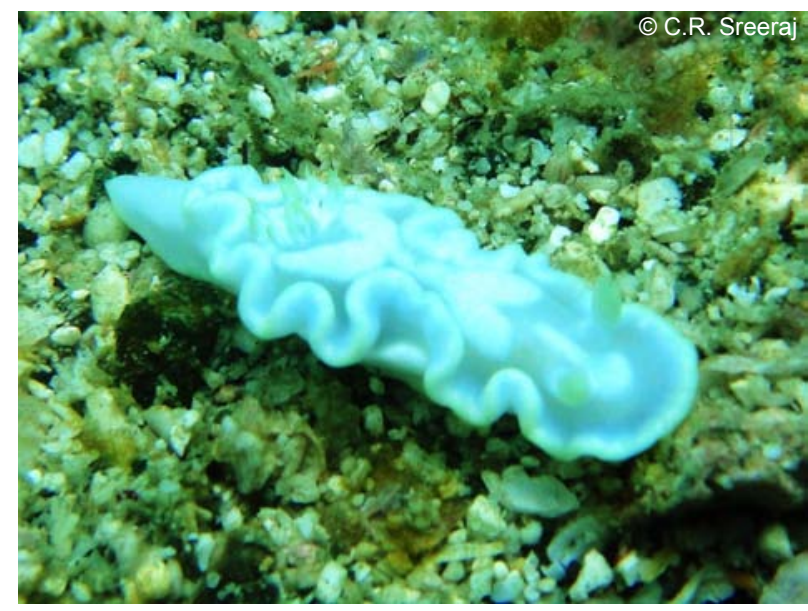

Image 5. Glossodoris pallida

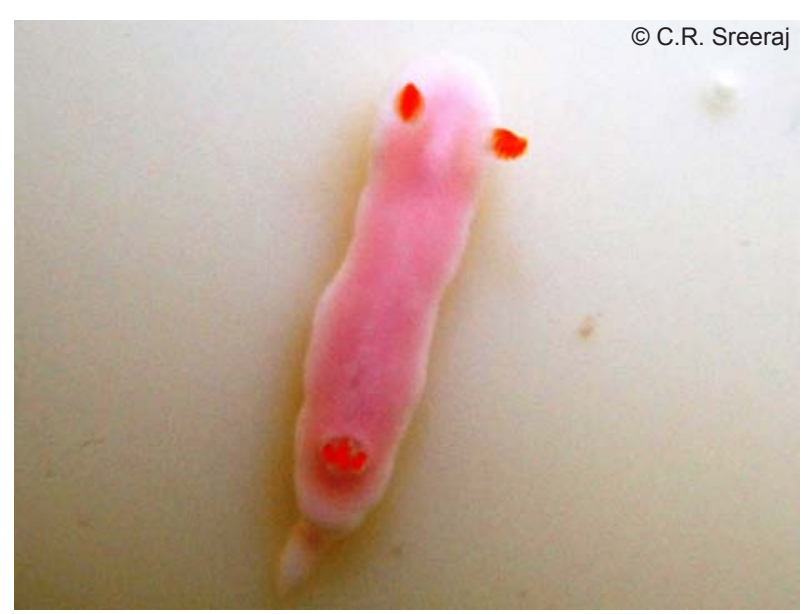

Image 6. Noumea simplex 
1850). Material examined: 20.ii.2011, one specimen, Kapang, Katchall Island. Identification: Body coloration highly variable, purple with a thin marginal white line. It has three longitudinal lines on the dorsum. The lines are dark blue and interrupted in the specimen observed in Katchall Island, instead of the yellow line seen in Pacific. The gills and rhinophores are orange (Image 7). Natural history: It was found in shallow reef where it was feeding on encrusting sponge. Distribution in India: Nicobar Islands, a new record from the present study. Remarks: New record to Indian Ocean.

30. Risbecia ghardaqana (Gohar \& Aboul-Ela, 1957). Material examined: 21.ii.2011, one specimen, Safed balu, Trinket Island. Distribution in India: Andaman Islands (Ramakrishna et al. 2010).

31. Risbecia pulchella (Rüppell \& Leuckart, 1828). Material examined: 22.ii.2011, two specimens, Kamorta Jetty. Distribution in India: Andaman Islands (Ramakrishna et al. 2010).

32. Thorunna australis (Risbec, 1928). Material examined: 16.ii.2011, one specimen, Champion, Nancowry Island; 20.ii.2011, four specimens, Kapang, Katchall Island. Distribution in India: Karnataka (Zacharia et al. 2008)

33. Thorunna florens (Baba, 1949). Material examined: 20.ii.2011, one specimen, Kapang, Katchall Island. Distribution in India: Andaman Islands.

34. Thorunna horologia Rudman, 1984. Material examined: 20.ii.2011, two specimens, Kapang, Katchall Island.

Distribution in India: Andaman Islands.

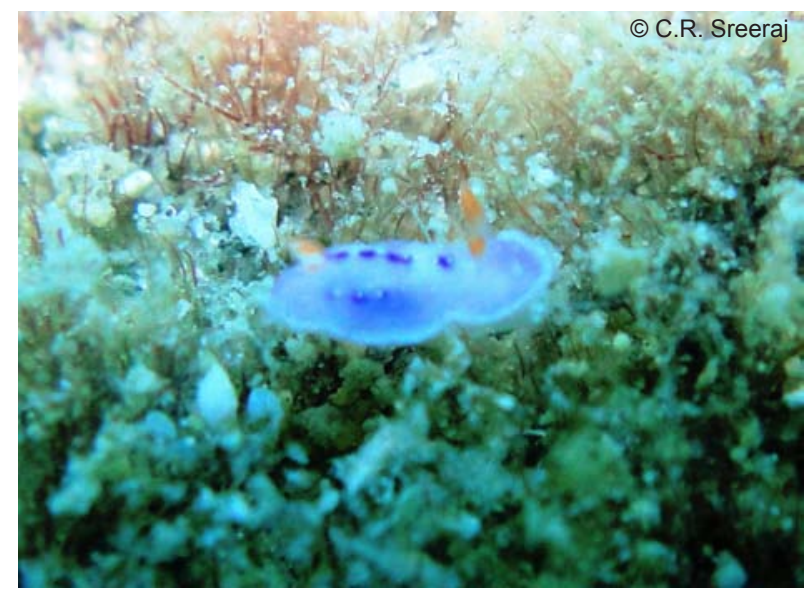

Image 7. Pectenodoris trilineata

\section{Phyllidiidae Rafinesque, 1814}

35. Phyllidia marindica (Yonow \& Hayward, 1991). Material examined: 13.ix.2010, one specimen, Campbell Bay, Great Nicobar; 16.ii.2011, one specimen, Champion, Nancowry Island; 19.ii.2011, one specimen, Kapila, Trinket Island; 21.ii.2011, two specimens, Safed balu, Trinket Island. Distribution in India: Andaman Islands, Lakshadweep Islands, Tamil Nadu (Apte 2009; Ramakrishna et al. 2010).

36. Phyllidia coelestis Bergh, 1905. Material examined: 17.ii.2011, one specimen, Kardip, Kamorta Island. Distribution in India: Andaman Islands, Lakshadweep Islands (Apte 2009; Ramakrishna et al. 2010).

37. Phyllidia elegans Bergh, 1869. Material examined: 19.ii.2011, one specimen, Kapila, Trinket Island. Identification: Body with black lines and spots. The tubercles are opaque white and the larger ones are tipped with yellow. The rhinophores are yellow. A thick median stripe of black on the sole of foot (Image 8). Natural history: Found on open reef slopes where it is frequently observed on the walls. Distribution in India: Nicobar Islands, a new record from the present study. Remarks: New record for Indian waters.

38. Phyllidia varicosa Lamarck, 1801. Material examined: 19.ix.2009, one specimen, Car Nicobar; 26.ii.2010, one specimen, Kamorta Island; 28.ii.2010, one specimen, Kamorta Island; 20.ii.2011, one specimen, Kapang, Katchall Island; 21.ii.2011, one specimen, Safed balu, Trinket Island; 22.ii.2011, two specimens, Kamorta Jetty. Distribution in India: Andaman Islands, Lakshadweep Islands, Tamil Nadu,

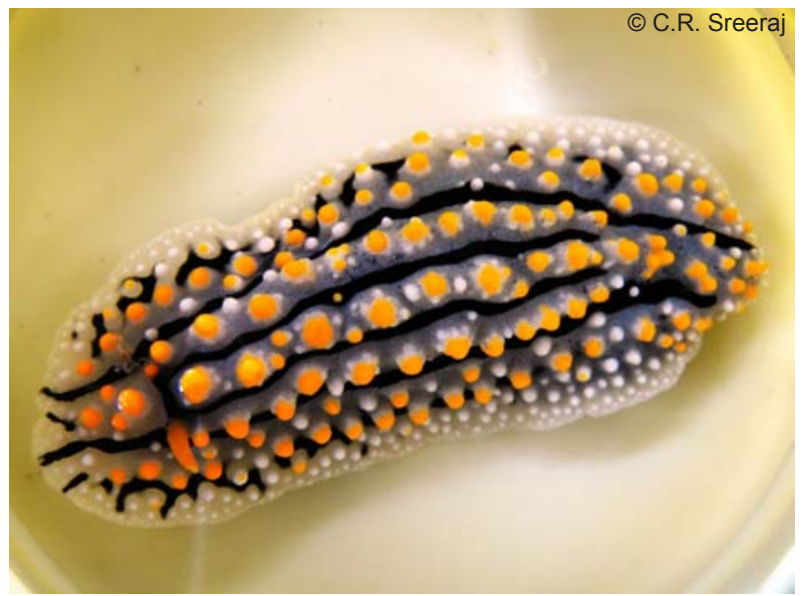

Image 8. Phyllidia elegans 
Karnataka (Rao et al. 1974; Zacharia et al. 2008; Apte 2009; Ramakrishna et al. 2010).

39. Phyllidiella pustulosa (Cuvier, 1804). Material examined: 22.ii.2011, one specimen, Kamorta Jetty. Distribution in India: Andaman Islands, Lakshadweep Islands (Apte 2009; Ramakrishna et al. 2010).

40. Phyllidiella rosans (Bergh, 1873). Material examined: 26.ii.2010, one specimen, Kamorta Island. Distribution in India: Andaman Islands, Lakshadweep Islands, Tamil Nadu (Apte 2009; Ramakrishna et al. 2010).

41. Phyllidiella rudmani Brunckhorst, 1993. Material examined: 13.xi.2010; three specimens, B. quarry, Campbell Bay, Great Nicobar; 19.xi.2009, one specimen, Car Nicobar; 19.ii.2011, one specimen, Kapila, Trinket Island; 20.ii.2011, one specimen, Kapang, Katchall Island; 22.ii.2011, one specimen, Kamorta Jetty. Distribution in India: Andaman Islands.

42. Phyllidiella zeylanica (Kelaart, 1859). Material examined: 19.ii.2011, six specimens, Kapila, Trinket Island; 20.ii.2011, two specimens, Kapang, Katchall Island; 21.ii.2011, one specimen, Safed balu, Trinket Island; 22.ii.2011, two specimens, Sanuh, Kamorta Island; 22.ii.2011, three specimens, Kamorta Jetty. Distribution in India: Andaman Islands, Lakshadweep Islands, Tamil Nadu, Karnataka, Andhra Pradesh, Gujarat (Rao et al. 1974; Eliot 1906; Narayanan 1968; Zacharia et al. 2008; Apte 2009; Ramakrishna et al. 2010).

43. Phyllidiopsis annae Brunckhorst, 1993. Material examined: 17.ii.2011, one specimen, Kardip, Kamorta Island. Identification: Body elongate with

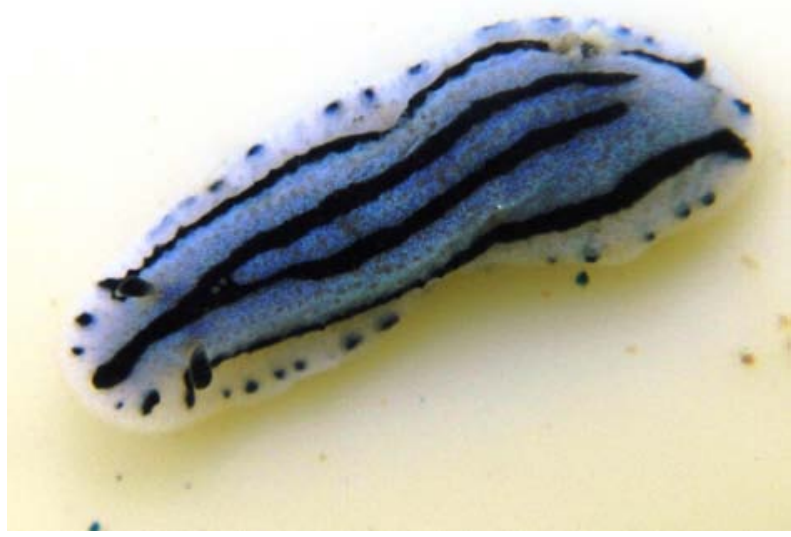

Image 9. Phyllidiopsis annae arrangement of four black lines as in $P$. striata. Mantle perimeter granulose with few tiny black spots. Rhinophores black (Image 9). Natural history: Found on shallow reef flat. Distribution in India: Nicobar Islands, a new record from the present study. Remarks: New record for Indian waters.

44. Phyllidiopsis gemmata (Pruvot-Fol, 1957). Material examined: 19.ii.2011, one specimen, Kapila, Trinket Island. Distribution in India: Lakshadweep Islands (Apte 2009).

45. Phyllidiopsis phiphiensis Brunckhorst, 1993. Material examined: 19.ii.2011, two specimens, Kapila, Trinket Island. Distribution in India: Andaman Islands, Lakshadweep Islands (Apte 2009; Ramakrishna et al. 2010).

46. Phyllidiopsis xishaensis Lin, 1983. Material examined: 19.ii.2011, one specimen, Kapila, Trinket Island. Distribution in India: Andaman Islands, Lakshadweep Islands (Apte 2009; Ramakrishna et al. 2010).

\section{Arminidae Iredale \& O'Donoghue, 1923}

47. Dermatobranchus rodmani Gosliner \& Fahey, 2011. Material examined: 18.ii.2011, two specimens, Alukiya, Kamorta Island. Identification: The body is elongated and triangular, flattened and narrow at posterior end. Notum is smooth and fleshy. Marginal sacs are visible along the mantle edge and each contains elongate, stiff rodlets. The color is pinkish-white. One or two transverse brown patches are present near the anterior and posterior thirds of the body. The notum and oral veil have a yellowish-margin, which contains fine brown spots. (Image 10). Natural history: Found

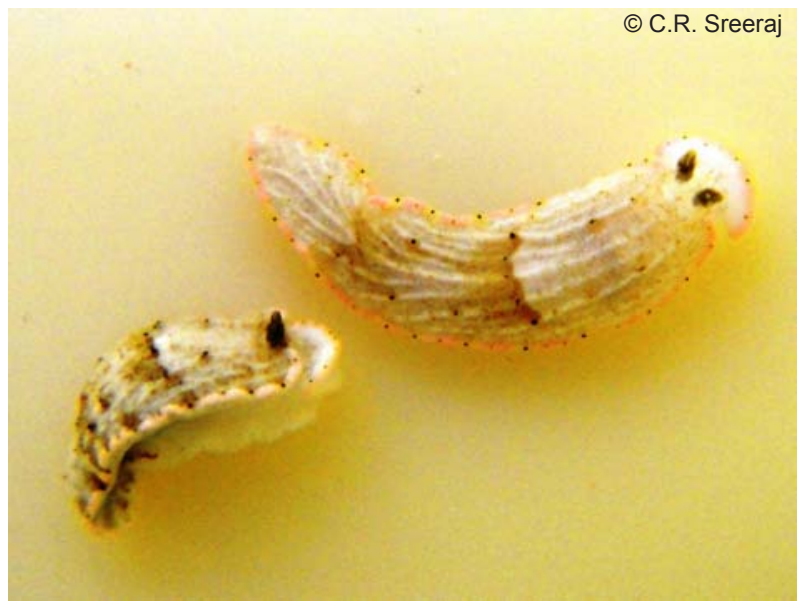

Image 10. Dermatobranchus rodmani 
on shallow patch reefs where they feed on soft corals.

Distribution in India: Nicobar Islands, a new record from the present study. Remarks: Previously known only from Madagascar and eastern Malaysia. This species was reported as Dermatobranchus sp. 16 in Gosliner et al. 2008 (page 313, bottom photo). This species is recorded for the first time after its description (Gosliner \& Fahey 2011) and is new record for Indian waters.

\section{Flabellinidae Bergh, 1881}

48. Flabellina bicolor (Kelaart, 1858). Material examined: 20.ii.2011, two specimens, Kapang, Katchall Island. Distribution in India: Lakshadweep Islands, Andhra Pradesh and Gujarat (Eliot 1906; Apte 2009; Apte et al. 2010).

49. Flabellina riwo Gosliner \& Willan, 1991. Material examined: 20.ii.2011, three specimens, Kapang, Katchall Island. Identification: The rhinophores are lamellate. The network of opaque white lines on the body and the sub apical band on the cerata are the distinctive characters (Image 11). Natural history: Found on shallow reef flats feeding on hydroids. Distribution in India: Nicobar Islands, a new record from the present study. Remarks: New record for Indian waters.

50. Flabellina rubrolineata (O’Donoghue, 1929). Material examined: 16.ii.2011, eight specimens, Champion, Nancowry Island; 19.ii.2011, eleven specimens, Kapila, Trinket Island. Distribution in India: Andaman Islands.

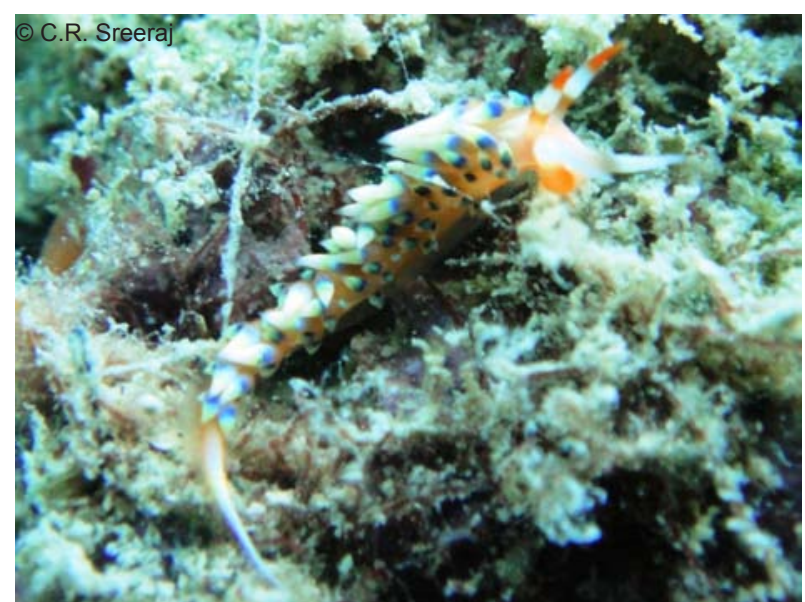

Images 12 \& 13. Phidiana indica.

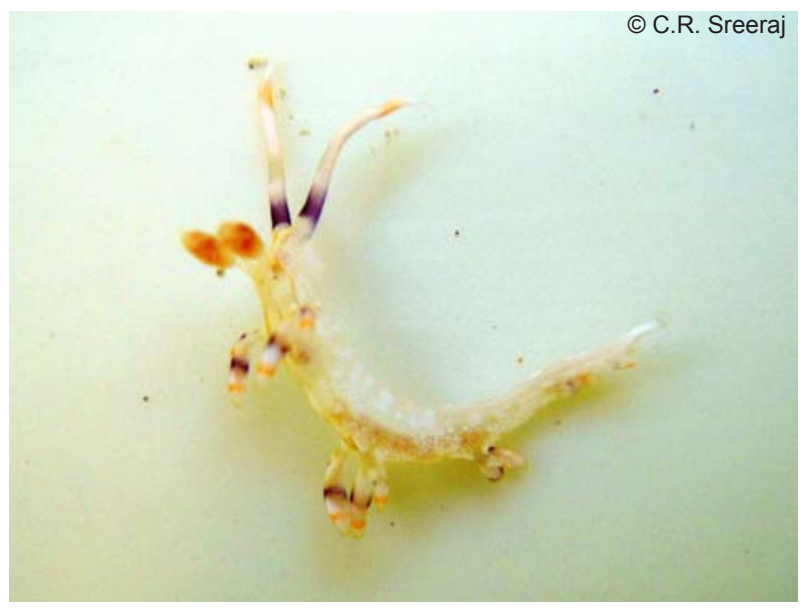

Image 11. Flabellina riwo

\section{Facelinidae Bergh, 1889}

51. Phidiana indica (Bergh, 1896). Material examined: 16.ii.2011, two specimens, Champion, Nancowry Island. 20.ii.2011, three specimens, Kapang, Katchall Island. Identification: Orange head with white and yellow markings and cerata with blue, yellow and red (Images 12 \& 13). Natural history: Found in shallow reefs feeding on hydroids. Distribution in India: Nicobar Islands, a new record from the present study. Remarks: New record for Indian waters

52. Pteraeolidia ianthina (Angas, 1864). Material examined: 26.ii.2011, one specimen, Kamorta Island; 16.ii.2011, one specimen, Champion, Nancowry Island. Distribution in India: Andaman Islands, Lakshadweep Islands, Gujarat, Tamil Nadu (Eliot 1909; Apte 2009; Apte et al. 2010; Ramakrishna et al. 2010).

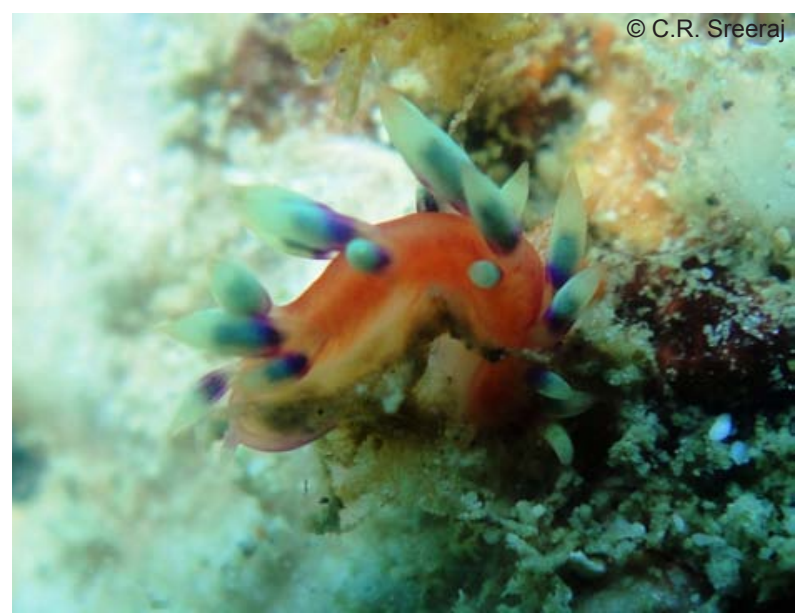




\section{Conclusion}

Most of the information available on the Indian opisthobranchs is pertaining to the peninsular coast. Opisthobranchiate taxonomy and ecology in Andaman and Nicobar Islands recently gained an attention. The present study represents the largest survey, in terms of geography and time from the coasts of Nicobar Islands. Nonetheless, due to an insufficient number of sampling dives, lack of dredge sampling as well as intertidal areas, the total number of the species was not as high as expected. The lack of night sampling is another factor limiting the quantity of identified species. Overall 52 opisthobranch species were recorded, of which 11 were new records for the Indian waters. These findings suggest that the Andaman and Nicobar Islands are indeed a region of high opisthobranch diversity, although till date it remains understudied as established by our new regional records. We expect to find as much as 400 species of opisthobranchs from the Nicobar group of Islands with focused work for a few years.

The opisthobranch fauna of this archipelago shows the affinity with species of neighboring countries bordering Andaman Sea rather than eastern coast of India. It is interesting to state that the opisthobranch species of Andaman have more similarity with that of Thai waters of Andaman Sea whereas that of Nicobar has more species similarity with Indonesian waters. However, the opisthobranch fauna of the oceanic Nicobar Islands have very less similarity with that of the opisthobranchs reported from the peninsular coast of India. In this context, the information about the opisthobranchs of Nicobar Islands adds value to the molluscan studies of Indian waters.

\section{REFERENCES}

Alder, J. \& A. Hancock (1864). Notice of a collection of nudibranchiate Mollusca made in India by Walter Elliot Esq., with descriptions of several new genera and species. Transactions of the Zoological Society of London 5: 113147

Apte, D. \& V.K. Salahuddin (2010). Record of Hexabranchus sanguineus (Rüppell \& Leuckart 1828) from Lakshadweep Archipelago, India. Journal of the Bombay Natural History Society 107(3): 261-262.

Apte, D., V. Bhave \& D. Parasharya (2010). An annotated and illustrated checklist of the opisthobranch fauna of Gulf of Kutch, Gujarat, India with 21 new records for Gujarat and 13 new records for India: part 1. Journal of the Bombay Natural History Society 107(1): 14-23.

Apte, D. (2009). Opisthobranch fauna of Lakshadweep Islands, India with 52 new records to Lakshadweep and 40 new records to India. Journal of the Bombay Natural History Society 106(2): 162-175.

Brunckhorst, D.J. (1993). The systematics and phylogeny of phyllidiid nudibranchs (Doridoidea). Records of the Australian Museum 16(Supplement): 1-107.

Dayrat, B. (2010). A monographic revision of basal discodorid sea slugs (Mollusca: Gastropoda: Nudibranchia: Doridina). Proceedings of the California Academy of Sciences Series 4, 61(1): 1-403.

Eliot, C. (1906). On the nudibranchs of south India and Ceylon, with special reference to the drawings by Kelaart and the collection belonging to Alder and Hancock preserved in the Hancock museum at New Castle-on-Tyne North. Proceedings of the Zoological Society of London, 1906 pt. II: 636-691 and 997-1008.

Eliot, C. (1910). Notes on nudibranchs from the Indian Museum. Records of the Indian Museum, 5(4): 247-252.

Eliot, C.N.E. (1909). Report on the nudibranchs collected by Mr. James Hornell at Okhamandal in Kattiawar in 19056. In: Report to the government of Baroda on the marine zoology of Okhamandal 1:137-145.

Fontana, A., C.L. Maria, L. D'souza, E. Mollo, G. Chandra, P.S. Naikk, Parameswaran, S. Wahidulla \& G. Cimino (2001). Selected chemo-ecological studies of marine opisthobranchs from Indian coasts. Journal of the Indian Institute of Science 81(4): 403-415.

Fontana, A., P. Cavaliere, N. Ungur, L. D'Souza, P. Parameswaram \& G. Cimino (1999). New scalaranes from the nudibranch Glossodoris atromarginata and its sponge prey. Journal of Natural Products 62: 1367-1370.

Gosliner, T.M. \& S.J. Fahey (2011). Previously undocumented diversity and abundance of cryptic species: a phylogenetic analysis of Indo-Pacific Arminidae Rafinesque, 1814 (Mollusca:Nudibranchia) with descriptions of 20 new species of Dermatobranchus. Zoological Journal of the Linnean Society 161: 245-356.

Gosliner, T.M., D.W. Behrens \& A. Valdes (2008). Indo Pacific Nudibranchs and Sea Slugs. Sea Challengers, 425pp.

Matwal, M. \& D. Joshi (2011). Record of Phyllidiella zeylanica (Mollusca:Gastropoda:Opisthobranchia) after 42 years from Gujarat, India. Journal of Threatened Taxa 3(7): 1951-1954.

Narayanan, K.R. (1968). On three opisthobranchs from the south-west coast of India. Journal of the Marine Biological Association of India 10(2): 377-380.

Narayanan, K.R. (1970). On two doridacean nudibranchs (Mollusca:Gastropoda) from the Gulf of Kutch, new to Indian coast. Advance Abstracts of Contributions on Fisheries and Aquatic Sciences in India 4(4): 313-314.

Raghunathan, C., C. Sivaperuman \& Ramakrishna (2010). An account of newly recorded five species of nudibranch 
(Opisthobranchia, Gastropoda) in Andaman and Nicobar Islands, pp 283-288. In: Recent Trends in Biodiversity of Andaman and Nicobar Islands. Zoological Survey of India, Kolkata.

Ramakrishna, C.R., C. Sreeraj, C. Raghunathan, J.S. Sivaperuman, R. Yogesh Kumar, Raghuraman, T. Immanuel \& P.T. Rajan (2010). Guide to Opisthobranchs of Andaman and Nicobar Islands. Zoological Survey of India, 196pp.

Rao, K.V. 1962 (For 1961). On two opisthobranchiate molluscs, Placobranchus ocellatus Hasselt and Discodoris boholiensis Bergh, from Indian waters not hitherto been recorded. Journal of the Marine Biological Association of India 3(1-2): 253-256.

Rao, K.V., P. Sivadas \& L.K. Kumari (1974). On three rare doridiform nudibranch molluscs from Kavaratti Lagoon, Laccadive Islands. Journal of the Marine Biological Association of India 16(1): 113-125.

Rudman,W.B.(1982). TheChromodorididae(Opisthobranchia: Mollusca) of the Indo-West Pacific: Chromodoris quadricolor, C. lineolata and Hypselodoris nigrolineata colour groups. Zoological Journal of the Linnaean Society 76: $183-241$.

Rudman,W.B.(1983). The Chromodorididae(Opisthobranchia: Mollusca) of the Indo-West Pacific: Chromodoris splendida, C. aspersa and Hypselodoris placida colour groups. Zoological Journal of the Linnaean Society 78: 105-173.

Rudman,W.B.(1984). The Chromodorididae(Opisthobranchia: Mollusca) of the Indo-West Pacific: a review of the genera. Zoological Journal of the Linnaean Society 81(2\&3): 115273.

Rudman,W.B.(1986). The Chromodorididae(Opisthobranchia: Mollusca) of the Indo-West Pacific: Noumea purpurea and
Chromodoris decora colour groups. Zoological Journal of the Linnaean Society 86: 309-353.

Rudman,W.B.(1995). The Chromodorididae(Opisthobranchia: Mollusca) of the Indo-West Pacific: further species from New Caledonia and the Noumea romeri group. Molluscan Research 16: 1-43.

Satyamurti, S.T. (1952). The Mollusca of Krusadai Island (in the Gulf of Mannar). Bulletin Madras Government Museum, New Series, Natural History Section 1(2) pt. 6, pp. 1-258, pls. 1-34.

Smith, E.A. (1878). On a collection of marine shells from the Andaman Islands. Proceedings of the Zoological Society of London 10: 804-821.

Sreeraj, C.R., P.T. Rajan., R. Raghuraman., C. Raghunathan., R. Rajkumar, Titus Immanuel \& Ramakrishna (2010). On some new records of sea slugs (Class: Gastropoda, Subclass: Opisthobranchia) from Andaman and Nicobar Islands, pp. 289-298. In: Recent Trends in Biodiversity of Andaman and Nicobar Islands. Zoological Survey of India, Kolkata.

Rao, N.V.S. (2003). Indian Seashells (Part-1) Polyplacophora and Gastropoda. Records of Zoological Survey of India. Z.S.I. Kolkata.

Rao, N.V.S. \& A. Dey (2000). Catalogue of marine molluscs of Andaman and Nicobar Islands. Records of the Zoological Survey of India, Occasional Paper No. 187, $\mathrm{x}+323 \mathrm{pp}$.

Zacharia, P.U., P.K. Krishnakumar, A.P. Dineshbabu, K. Vijayakumaran, P. Rohit, S. Thomas, G. Sasikumar, P. Kaladharan, R.N. Durgekar \& K.S. Mohamed (2008). Species assemblage in the coral reef ecosystem of Netrani Island off Karnataka along the southwest coast of India. Journal of the Marine Biological Association of India 50(1): 87-97. 